HID 43 (2016)

\title{
DE MALLORCA A NÁPOLES, LLEIDA Y ROMA: LOS VIAJES DEL CANÓNIGO GABRIEL CERDÀ (1450-1491)*
}

\author{
FROM MAJORCA TO NAPLES, LLEIDA AND ROME: THE VOYAGES \\ OF THE CANON GABRIEL CERDÀ (1450-1491)
}

\author{
Albert Cassanyes Roig \\ Universitat de Lleida \\ albert.cassanyes@historia.udl.cat
}

RESUMEN: Entre los distintos grupos de viajeros de la Edad Media, los eclesiásticos fueron uno de los más activos. Los canónigos mallorquines de la segunda mitad del siglo XV también surcaron los mares para llegar a diferentes destinos. A través del presente artículo se estudian los viajes realizados por uno de ellos, Gabriel Cerdà, que se dirigió a los principales centros de poder de la Corona de Aragón (Nápoles y Catalunya) y de la Iglesia (Roma), aunque también frecuentó la ciudad de Lleida para estudiar y ostentar otro canonicato en su catedral. Su caso es un paradigma del comportamiento de los canónigos mallorquines en relación a su movilidad geográfica.

PalabRaS ClaVE: Canónigos; Cerdà, Gabriel; Lleida; Mallorca; rentas; viajes.

ABSTRACT: Among the different groups of medieval travellers, ecclesiastics were one of the most active. Fifteenth-century Majorcan canons, too, crossed the seas to reach their destinations. This paper considers the voyages of one of them, Gabriel Cerdà, who went to the most important centres of power of the Crown of Aragon (Naples and Catalonia) and of the Church (Rome), although he also frequented Lleida in order to study and to hold another canonry in its cathedral. His case is a paradigm of Majorcan canons' performance related to their geographical mobility.

KEYWORDS: Canons; Cerdà, Gabriel; Lleida; Majorca; incomes; voyages.

* El presente artículo ha sido elaborado con el apoyo de la Secretaria d'Universitats i Recerca del Departament d'Economia i Coneixement de la Generalitat de Catalunya. Abreviaturas utilizadas: ACL $=$ Archivo Capitular de Lleida; ACM = Archivo Capitular de Mallorca. 
El hombre medieval se movía. A pesar de los riesgos y peligros, recorría las vías terrestres y surcaba los mares. Sus objetivos eran muy variados, y alcanzaban desde la resolución de ciertas cuestiones en los centros de poder hasta la obtención de beneficios económicos a través de la práctica del comercio ${ }^{1}$ o la participación en empresas guerreras ${ }^{2}$. Tampoco hay que olvidar la potente motivación piadosa de las peregrinaciones que perseguían la salvación del alma tras un penoso trayecto hasta los Santos Lugares ${ }^{3}$. Así pues, viajaban personas de todos los estamentos, cada una con sus propias razones. Uno de los colectivos que presentó una mayor movilidad geográfica fue el de los eclesiásticos. Muchos de ellos eran peregrinos que se desplazaban con objetivos espirituales. Pero también hubo procuradores de órdenes religiosas o miembros de la alta jerarquía eclesiástica que viajaban de un lugar a otro para ocuparse de asuntos temporales o para visitar los claustros y territorios que estaban bajo su autoridad. Tampoco hay que olvidarse de los clérigos estudiantes, entre los cuales se hallaban los controvertidos goliardos ${ }^{4}$.

El presente artículo pretende acercarse a la movilidad de un grupo eclesiástico concreto en una cronología bajomedieval específica: los canónigos de Mallorca durante la segunda mitad del siglo XV. En ocasiones las fuentes indican que los eclesiásticos viajaban como pasajeros en las embarcaciones que se dirigían a la isla, ya fuera esta su destino final o solo un punto de escala. Por ejemplo, el obispo Pedro de Santángel (1465-1466) llegó a su nueva diócesis a bordo de dos galeazas de florentinos ${ }^{5}$. En este sentido, algunos de los eclesiásticos que presentaban una mayor movilidad fueron los canónigos que conformaban el cabildo catedralicio de Mallorca, cuyos viajes los llevaron a los diferentes territorios de la cuenca mediterránea occidental. A tal efecto, se ha querido tomar como modelo el canónigo Gabriel Cerdà. El suyo es un caso paradigmático y altamente ilustrativo, ya que, además de ser un personaje relativamente bien conocido -como mínimo en sus grandes rasgos biográficos ${ }^{6}$-, ejerció su canonicato íntegramente durante la segunda mitad del siglo XV (1450-14917 $)$, de modo que se conserva la serie documental referente al pago de las distribuciones cotidianas de forma casi completa, como se indicará. Así pues, este canónigo resulta especialmente útil para alcanzar el objetivo de este artículo, aunque es evidente que su caso no se podrá generalizar hasta que no se realicen ulteriores investigaciones ${ }^{8}$.

1. El comercio medieval es una de las cuestiones que cuenta con más bibliografía, tanto general como centrada en el espacio regional, por lo que es imposible ofrecer un listado exhaustivo. Véase, a modo de ejemplo, la clásica obra de Postan 1978; Ruzafa García 1994; Igual Luis 1997.

2. Especialmente, las Cruzadas. Oldenbourg 1965.

3. Véase, por ejemplo, Alvira Cabrer 1999; Novoa 2003; Andrade Cernadas 2014.

4. La movilidad geográfica de los eclesiásticos en la Edad Media es un tema que requiere de un mayor ahondamiento. Es interesante la breve aproximación realizada por Rapp 1973, pp. 201-205.

5. ACM, Mensa Capitular, 2770, f. 34r.

6. Véanse Mas i Forners 2000; Barceló Crespí, Ensenyat Pujol 2013, pp. 20-22.

7. Antoni Salvador Cerdà hizo inventario de los bienes de su tío-abuelo Gabriel Cerdà el 2 de diciembre de 1491. Mas i Forners 2000, p. 442.

8. Los estudios sobre la movilidad de clérigos durante la Edad Media son todavía escasos y, de momento, no hay obras específicas sobre esta cuestión, aunque algunos autores la han tratado, ni que 
La principal fuente utilizada para la elaboración de este trabajo ha sido la serie documental "Mensa capitular", conservada en el Archivo Capitular de Mallorca. Las ausencias de los canónigos tenían repercusiones a nivel económico que afectaban los propios prebendados. Los libros de la "Mensa Capitular", que cuentan con un apartado destinado a contabilizar los pagos de las pensiones a los canónigos, ya se preocupan de distinguir claramente los que estaban presentes en Mallorca de los que se hallaban ausentes. Esto se debe a que los canónigos cobraban dos partidas económicas anuales ${ }^{9}$ diferentes. En primer lugar, percibían una pensión, la denominada "porción canonical", que era una cantidad fija -o la parte proporcional en el caso de los canónigos que tomaban posesión del canonicato una vez iniciada la anualidad, o que morían en el transcurso de esta- que se fue incrementando a lo largo del tiempo ${ }^{10}$ y que cobraban por el simple hecho de ostentar la prebenda. Esta cantidad era abonada frecuentemente a todos los canónigos, tanto a los presentes como a los ausentes, aunque, entre estos últimos, no es raro hallar porciones canonicales más reducidas o retrasos en los pagos. En segundo lugar, percibían las llamadas "distribuciones cotidianas", un pago anual cuyo monto dependía del tiempo que los canónigos hubieran pasado en Mallorca. Así pues, los prebendados cobraban unos cuatro sueldos por cada día que hubieran estado presentes en la isla, de modo que al final del año, en el caso que no hubieran salido de Mallorca, percibían un total de setenta y tres libras. Gracias a las distribuciones cotidianas, es posible determinar no solo las ausencias de los canónigos, sino también la duración de sus viajes. Además, en ocasiones se indica en el margen el origen o el destino de sus trayectos, lo que permite intentar reconstruir sus movimientos a lo largo del mar.

De hecho, vivir en una isla no significaba vivir aislado. Precisamente, a lo largo de la Edad Media, las islas, en general, estaban mejor conectadas que ciertos pueblos del interior continental, puesto que las comunicaciones por vía marítima resultaban más rápidas y baratas que no aquellas que transcurrían por caminos y carreteras ${ }^{11}$. El mar Mediterráneo constituía el centro del mundo medieval occidental, ejerciendo tanto de frontera entre las dos grandes religiones monoteístas como de vía de contacto entre todos los pueblos que se asomaban a sus orillas ${ }^{12}$. Además, a través del estrecho de Gibraltar las naves llegaban hasta las costas del mar del Norte, Inglaterra y las ciudades hanseáticas, mientras desde los puertos

sea de forma parcial, en otras investigaciones. En cambio, la historia de las migraciones cuenta con una mayor literatura, incluso para el caso de Mallorca. Vaquer Bennàssar 1995, 1999.

9. A efectos del pago de las pensiones a los canónigos, se consideraba que el año se iniciaba el primero de abril y que finalizaba el último día de marzo del año inmediatamente siguiente. Así pues, por ejemplo, en el caso del libro de la Mensa Capitular correspondiente al año 1450, se debe considerar que los pagos abrazan el período comprendido entre el $1^{\circ}$ de abril de 1450 y el 31 de marzo de 1451. Este principio es el que se ha seguido a la hora de resolver las datas cronológicas de las fuentes documentales.

10. Por ejemplo, en 1455 los canónigos mallorquines percibían una porción canonical de 50 libras. ACM, Mensa Capitular, 2762, ff. 49r-52v. En 1472 había sido aumentada hasta las 60 libras. ACM, Mensa Capitular, 2776, ff. 30r-31v.

11. Igual Luis 2006-2008, pp. 192-193.

12. Tangheroni 1996. 
mediterráneos orientales salían las rutas terrestres, la más importante de las cuales fue la Ruta de la Seda, que permitían alcanzar los lejanos territorios del Extremo Oriente ${ }^{13}$.

De forma más concreta, Mallorca se veía beneficiada por su posición geográfica de encrucijada ${ }^{14}$, situada en medio del Mediterráneo occidental, siendo un punto de escala casi obligado tanto en los viajes entre los mercados cristianos del norte y los musulmanes del sur ${ }^{15}$ como en los trayectos de las naves genovesas e italianas en general que, cruzando el estrecho de Gibraltar, alcanzaban los puertos atlánticos ${ }^{16}$. Pero también formaba parte de la denominada "Ruta de las Islas", que unía la península Ibérica con Italia ${ }^{17}$. Igualmente, Mallorca se especializó en el comercio de redistribución y se convirtió en la base a la cual llegaban los productos exóticos que luego eran vendidos en los mercados del Levante peninsular, especialmente Valencia ${ }^{18}$, aunque también Barcelona ${ }^{19}$. Esto no implica, pero, que los mallorquines no aprovechasen estas circunstancias para colocar su propio género en los mercados o que no tuvieran relación con otros puertos mediterráneos. Más bien al contrario, los mercaderes insulares llevaban a cabo una intensa actividad comercial. Su principal mercado era el norte de África, desde donde se importaban trigo, lana, carne y oro, y se exportaban paños. Asimismo, los mercados italianos eran muy significativos, sobre todo Génova, Nápoles ${ }^{20}$, Sicilia y Cerdeña ${ }^{21}$, pero también Milán, Florencia y Venecia. Los puertos de Constantinopla, Rodas y Alejandría, en el Mediterráneo oriental, fueron visitados frecuentemente por los mallorquines, que incluso llegaron al otro extremo del continente europeo, a Inglaterra y Flandes ${ }^{22}$.

13. La conquista de Constantinopla por parte de los turcos en 1453 supuso el cierre de la Ruta de la Seda y la búsqueda de nuevas vías marítimas para llegar a Oriente.

14. Salicrú i Lluch 2008, pp. 115-116.

15. Para las relaciones comerciales entre Mallorca y el norte de África, véanse López 2004; Beltran Perelló 2008.

16. Cateura Bennàsser 2000, p. 86.

17. Villanueva Morte 2008, p. 37.

18. Guiral-Hadziiossif 1989.

19. Vaquer Bennàssar 1997, pp. 148-149.

20. Barceló Crespí, Ensenyat Pujol 2000, p. 27.

21. Los estudios sobre el comercio sardo realizados por historiadores italianos son muy abundantes. A modo de ejemplo pueden citarse Meloni 1977; Simbula 1993; Tognetti 2005.

22. Urgell Hernández 2000. Existen muchos trabajos publicados sobre el comercio mallorquín, y este no es el lugar para exponer una bibliografía exhaustiva. Algunos trabajos destacables son Sevillano Colom 1968-1972; Macaire 1986; Vaquer Bennàssar 1991, 2001. Sobre las relaciones entre Mallorca y el mundo atlántico, aunque para los siglos XIII y XIV, véase, a modo de ejemplo, Ortega Villoslada 2008, 2013. 


\section{El Doblemente CANÓNigo Gabriel CERDÀ}

Gabriel Cerdà fue el máximo exponente de la saga de los Cerdà de Binicalvell $^{23}$ en la Iglesia mallorquina durante la segunda mitad del siglo XV. Seguramente nació en la villa de Santa Margalida, y era sobrino de Antoni Cerdà ${ }^{24}$, que fue, entre muchos otros cargos eclesiásticos, arzobispo de Mesina (1447-1449), cardenal presbítero del título de San Crisógono (1448-1459) y obispo de Lleida (1449-1459). Reconocido como una persona de gran inteligencia y prudencia ${ }^{25}$, Gabriel Cerdà se doctoró en Cánones ${ }^{26}$, muy probablemente en el Estudio General de Lleida ${ }^{27}$. Asimismo, el joven Gabriel Cerdà inició en esta ciudad su carrera eclesiástica, pues fue nombrado canónigo de la sede leridana el 17 de diciembre de $1449^{28}$ y tomo posesión personalmente de la misma el 20 de diciembre de $1451^{29}$. Esta prebenda se vio completada poco después con su nombramiento como sochantre y responsable de la distribución de las horas, además de ejercer de prepósito de la pavordía del mes de mayo ${ }^{30}$ desde septiembre de $1450^{31}$. Todos estos cargos los recibió siendo su tío Antoni Cerdà obispo de Lleida, en una política de favorecimiento que no ocultaba la práctica del nepotismo. Más bien al contrario, en el acta capitular en la que se recoge el nombramiento de Gabriel Cerdà como prepósito de mayo, se menciona Gabrieli Cerdà, consanguineo dicti Reverendissimi domini cardinalis Episcopi Ilerdensis, esto es la existencia de un parentesco entre el canónigo y el obispo ${ }^{32}$ que, en otras ocasiones, aparece explicitado en el grado, poco específico, de nepos ${ }^{33}$. Tampoco sería extraño que el nombramiento se hubiera planificado desde Roma, puesto que la documentación remitida al cabildo leridano fue gestionada por Joan Margarit i Pau ${ }^{34}$, que obtuvo la púrpura en 1483, y por Pere Eiximenis, que ejercía de secretario del cardenal Cerdà. Sea como sea, Gabriel Cerdà contaba con dispensa de residencia y, de acuerdo con las

23. Sobre los Cerdà de Binicalvell, véase Mas i Forners 2000.

24. Su ascendencia, pero, no es clara. Según March y Rosselló Vaquer, era hijo de Esteve Cerdà, hermano del cardenal Antoni Cerdà. March Ques, Rosselló Vaquer 1981, p. 185.

25. March Ques, Rosselló Vaquer 1981, p. 185.

26. "Gabriel Cerdà, decretorum doctor". ACL, AC_0053 (Actas Capitulares, 1475-1481), f. 64r.

27. Otros autores afirman que obtuvo el grado de doctor en Teología. Mas i Forners 2000, p. 439 Aun así, no se tiene constancia documental de estos presuntos estudios teológicos.

28. ACL, AC_0049 (Actas Capitulares, 1448-1450), f. 122rv. En esta ocasión, su procurador, Antoni Pere, tomó posesión de la prebenda en nombre de Gabriel Cerdà.

29. Su toma de posesión en ACL, AC_0050 (Actas Capitulares, 1451-1456), f. 55v.

30. El prepósito o pavorde administraba una serie de bienes patrimoniales. Por norma general, en los cabildos había doce pavordes, uno para cada mes del año. El cargo recaía en un canónigo que tenía que distribuir entre los demás las porciones canonicales anuales en doce mensualidades. La prepositura o pavordía de mayo de Lleida recibía los productos de varios términos pertenecientes, mayoritariamente, a la actual comarca catalana de les Garrigues, como les Borges Blanques, Sudanell o Montoliu, entre otras. A pesar de ser la pavordía que ocupaba una mayor extensión geográfica, presentaba unas condiciones pluviométricas áridas, con escasez de lluvias durante todo el año. Argilés 2010, p. 180-183.

31. ACL, AC_0049 (Actas Capitulares, 1448-1450), ff. 169v-171r.

32. ACL, AC_0049 (Actas Capitulares, 1448-1450), f. 170r.

33. Por ejemplo, en ACL, AC_0050 (Actas Capitulares, 1451-1456), f. 11v.

34. Sobre este personaje, Tate 1955 . 
constituciones de la Iglesia de Lleida de 1237, los veinticinco canónigos que conformaban el capítulo tenían derecho a percibir una porción, estuvieran presentes o ausentes ${ }^{35}$. A pesar de ello, Cerdà asistió con una cierta asiduidad a las sesiones capitulares leridanas. Sin embargo, su presencia no era regular, sino que alternaba períodos. A veces era porque el canónigo no se hallaba en aquel momento en Lleida. En otras ocasiones, pero, se iba antes de que acabaran las sesiones. Así aparece indicado en el acta de la sesión del 7 de abril de 1455, en la cual el cabildo instó a los prepósitos a observar las constituciones y costumbres de la Iglesia leridana; una breve nota final indica que trattando de dicto negotio, dominos decanus et $\mathrm{Ga}$ briell Cerdà exierunt capituli et non fuerunt in conclusione predicte ordinationis ${ }^{36}$.

Al mismo tiempo que su canonicato leridano, Gabriel Cerdà obtuvo una prebenda en la catedral de Mallorca: su nombramiento tuvo lugar el 15 de agosto de 1450 , cuando fue elegido para ocupar la canonjía que había quedado vacante por muerte de Bernat Berard ${ }^{37}$. Asimismo, en 1454 se menciona que Cerdà ostentaba la dignidad de sacrista del cabildo catedralicio mallorquín ${ }^{38}$. Finalmente, fue también vicario general de la diócesis de Mallorca bajo el pontificado del obispo Rodrigo de Borja (1489-1492), futuro papa Alejandro VI.

Gabriel Cerdà falleció en $1491^{39}$. El 4 de diciembre de 1491 la noticia de su muerte había llegado ya a Lleida, porque el beneficiado Joan de Castres, ejerciendo de procurador de Felipe de Aviñón, presentó al cabildo catedralicio una carta apostólica por la que se autorizaba a este último a ocupar el canonicato y la prepositura vacantes por muerte de Gabriel Cerdà ${ }^{40}$. Otro candidato a ostentar estos mismos cargos fue Francesc Nicolau, presbítero valenciano y procurador del vicecanciller de Rodrigo de Borja, entonces cardenal-obispo de la diócesis portuense. El cabildo catedralicio de Lleida, en sesión de 7 de diciembre de 1491, optó por conceder el canonicato vacante a Francesc Nicolau ${ }^{41}$.

\section{Los Viajes del CANÓNigo GABRIEl CeRdÀ}

Gabriel Cerdà fue canónigo durante cuarenta años. Además, hay que destacar que ostentó dos canonjías al mismo tiempo en dos cabildos catedralicios diferentes, en Lleida y en Mallorca. A pesar de disfrutar del privilegio de ausencia -el denominado "privilegio de fructibus in absentia"-, sus obligaciones canonicales lo llevaron a viajar continuamente entre las dos sedes episcopales para resolver de forma personal ciertos asuntos. Sin embargo, Gabriel Cerdà también visitó otras

35. Piqué Badia 2002-2003, p. 432.

36. ACL, AC_0050 (Actas Capitulares, 1451-1456), f. 208v.

37. ACM, Mensa Capitular, 2758, f. 55v. Tomó posesión del canonicato dos días después, el 17 de agosto. ACM, 15576 (Libro de Posesorios), f. 55r.

38. ACM, Mensa Capitular, 2761, f. 42v.

39. March y Rosselló Vaquer afirman que murió en septiembre de 1490. March Ques, Rosselló Vaquer 1981, p. 185.

40. ACL, AC 0055 (Actas Capitulares, 1489-1497), f. 105rv.

41. ACL, AC_0055 (Actas Capitulares, 1489-1497), f. 105v-106v. 
ciudades mediterráneas, sin que se pueda precisar el motivo de tales estancias. Aun así, no se puede descartar una cierta influencia de su tío, el cardenal Antoni Cerdà, ya que buena parte de los destinos están vinculados a este personaje o a otros centros de poder de la Corona de Aragón. En cualquier caso, muchos de sus viajes han quedado reflejados en los libros de la Mensa Capitular de Mallorca, indicándose, en varias ocasiones, el destino de sus trayectos, o su procedencia, según el caso. Esto ha permitido reconstruir la movilidad de Gabriel Cerdà durante toda la segunda mitad del siglo XV.

La ciudad de Nápoles fue el destino del primer viaje realizado por Gabriel Cerdà como canónigo de Mallorca; se embarcó en una nave en dirección al sur de la península Itálica el 15 de febrero de $1451^{42}$, esto es exactamente seis meses después de haber tomado posesión de su prebenda mallorquina. Se ignora cuánto tiempo estuvo en Nápoles, ya que cuando vuelve a aparecer en los registros, el 10 de agosto de 1453, no se indica desde qué puerto retornó a Mallorca ${ }^{43}$; en cualquier caso, no es probable que regresara desde Italia, porque Cerdà se encontraba en Lleida entre el 14 de agosto de 1452 y el 2 de julio de 1453 . No hay que olvidar que la ciudad de Nápoles fue uno de los principales destinos hacia donde se dirigían los canónigos, y buena parte de los diplomáticos, durante los años centrales del siglo XV, porque en ella residía la corte del rey Alfonso el Magnánimo desde la conquista de la misma en 1442. Además, el obispo Juan García, titular de la diócesis mallorquina entre 1447 y 1459, fue consejero y confesor del Magnánimo ${ }^{44}$, de modo que su presencia en la corte napolitana fue obligada hasta la muerte del monarca ${ }^{45}$. Por tanto, es posible que Gabriel Cerdà se dirigiera a Nápoles para resolver algún encargo mandado por el cabildo catedralicio de Mallorca, siendo como era esta ciudad el principal centro del poder monárquico de la Corona de Aragón ${ }^{46}$. Tampoco se puede descartar una intervención de su tío, el cardenal Antoni Cerdà, que habría estado muy vinculado a la corte del Magnánimo, primero como embajador pontificio de Eugenio IV y luego como preceptor de los hijos del monarca $^{47}$. Quizá Gabriel Cerdà viajó a Nápoles para entrevistarse con el purpurado, que pasaba algunas temporadas en esta ciudad.

Poco después de su regreso a Mallorca en agosto de 1453, Gabriel Cerdà volvió a embarcarse en un viaje que lo mantuvo alejado de la isla durante poco más de cuatro años: salió de Mallorca el 28 de noviembre de $1453^{48}$ y no retornó hasta el 7

42. ACM, Mensa Capitular, 2758, f. 55v.

43. ACM, Mensa Capitular, 2760, f. 44v.

44. Furió 1852, pp. 254-255.

45. Tras el fallecimiento del rey, la división del efímero Reino de las Dos Sicilias supondría la separación política de Nápoles y Mallorca, ya que el primero fue heredado por el hijo natural del Magnánimo, Fernando I de Nápoles, mientras Mallorca, junto al resto de territorios de la Corona de Aragón, pasó al hermano del rey difunto, Juan II.

46. Las embajadas a la corte napolitana de Alfonso el Magnánimo por parte de mallorquines fueron muy frecuentes a causa de la inexistencia de unas cortes propias. Barceló Crespí, Ensenyat Pujol 2000, p. 59.

47. Bover de Rosselló 1838, p. 72.

48. ACM, Mensa Capitular, 2760, f. 44v. 
de marzo de $1458^{49}$. Durante este período visitó la ciudad de Lleida en numerosas ocasiones y se estableció en ella durante algunos meses. Además, se lo documenta en Valencia en 1455, pues los jurados de la villa de Santa Margalida, en Mallorca, le mandaron una carta en esta ciudad el 21 de noviembre de aquel año ${ }^{50}$. Como Cerdà es hallado en Lleida tanto antes como después de esta fecha, parece ser que el viaje a Valencia fue, más bien, un hecho puntual y una etapa más del periplo, no el destino efectivo de este. Quizá la causa de la visita valenciana deba buscarse en un encargo para resolver algún asunto relacionado con el nuevo papa Calixto III -conocido como Alfonso de Borja con anterioridad a su ascenso al solio pontifico, oriundo del Reino de Valencia y amigo personal del cardenal Antoni Cerdà-, que había sido elegido para la tiara pontificia en abril de aquel mismo año ${ }^{51}$. Por otro lado, durante esta ausencia Gabriel Cerdà también participó en las Cortes de Barcelona reunidas entre los años 1454 y 1458. El mallorquín tomó parte en estas como representante del obispo de Lleida, su tío Antoni Cerdà, representación que compartió con el canónigo Berenguer de Sos a partir de $1455^{52}$.

Lleida, en cambio, sí que fue un destino habitual de Gabriel Cerdà, que, como ya se ha indicado, había sino nombrado canónigo de su cabildo catedralicio en 1449. Así pues, Cerdà aprovechaba sus viajes para realizar una escala en Lleida, residir unos meses en la ciudad y asistir a las sesiones capitulares. Pero la ciudad del Segre era también muy importante por su Estudio General. Es posible que Cerdà quisiera cursar estudios superiores para progresar en su carrera eclesiástica y que, por este motivo, se matriculara en una universidad cuando accedió al canonicato ${ }^{53}$. El centro elegido sería el Estudio General de Lleida, centro en el cual su tío habría sido estudiante y catedrático ${ }^{54}$, y situado, precisamente, en la ciudad de la cual era obispo. Lleida fue un destino especialmente importante para los mallorquines en general, y para los canónigos en particular, que se dirigían a su Estudio General, donde, preferentemente, obtenían el grado de doctor en Derecho Canónico o en Derecho Civil ${ }^{55}$. En este sentido, Cerdà no fue una excepción, ya que, como se ha indicado anteriormente, consiguió el grado de doctor en Cáno-

49. ACM, Mensa Capitular, 2763, f. 41r.

50. Barceló Crespí, Ensenyat Pujol 2013, p. 22, nota 25.

51. Para el personaje de Calixto III son especialmente recomendables los estudios de Miguel Navarro Sorní. Una breve biografía del papa elaborada por este autor es Navarro Sorní 2005.

52. Martí Sentañes 2008, pp. 132-133. La autora indica, erróneamente, que Gabriel Cerdà quizá fuera un hermano del obispo.

53. En una acta capitular de 1452 se refieren los estudios de los diferentes canónigos leridanos. Gabriel Cerdà no contaba todavía con ningún grado académico. ACL, AC_0050 (Actas Capitulares, 1451-1456), f. 95r.

54. Bover de Rosselló 1838, pp. 70-71.

55. Lladonosa indica que los estudiantes de Leyes y Cánones mallorquines preferían trasladarse a Lleida por el prestigio que su Estudio General tenía en estas disciplinas. Lladonosa i Pujol 1976, pp. 50-51. Sin embargo, los estudiantes mallorquines (y buena parte de los procedentes de la Corona de Aragón) optaban por cursar sus estudios en las universidades italianas, principalmente Boloña en el siglo XIV y Pisa-Florencia en el período del tránsito a la modernidad. Véase Ramis Barceló 2014, pp. 40-47. 
nes, aunque no se puede precisar en qué universidad se le colacionó el título ${ }^{56}$. Igualmente, según Lladonosa, Cerdà fue uno de los acusados en el proceso que se siguió contra una serie de estudiantes sublevados el día 11 de junio de 1456 a raíz de la detención del estudiante Jaume $\mathrm{Mas}^{57}$. También el mismo autor indica que Cerdà fue uno de los becarios del Colegio de la Asunción ${ }^{58}$.

Con posterioridad a este largo viaje de cuatro años, Cerdà continuó visitando Lleida de forma bastante asidua. Durante la Guerra Civil Catalana (1462-1472) no se encontraba en la ciudad, que fue sitiada por las tropas de Juan II desde finales de abril de 1464 y que no capituló hasta el 6 de julio siguiente ${ }^{59}$. Entre el 8 de abril de $1464^{60}$ y el 13 de mayo de $1465^{61}$ Gabriel Cerdà realizó un viaje cuyo destino se ignora. Sin embargo, sí que estuvo en la ciudad de Lleida a partir del día 1 de agosto de 1464 -casi un mes después de levantado el asedio-; precisamente el 3 de agosto de aquel año el cabildo catedralicio trató sobre un compromiso entre la institución canonical y el canónigo Cerdà, además de Lluis Ventosa, sobre la prepositura del mes de mayo ${ }^{62}$. No se puede descartar que Cerdà aprovechara el viaje para pararse momentáneamente en Lleida para resolver aquel asunto, puesto que fue tratado solo dos días después de su llegada, aunque no abandonó la ciudad hasta el 30 de octubre siguiente ${ }^{63}$.

En una ocasión se indica que Gabriel Cerdà viajó a Catalunya, aunque no se especifica su destino concreto. El Principado era uno de los mayores centros de poder de la Corona de Aragón, especialmente tras la muerte del rey Alfonso el Magnánimo y el retorno de la corte a Barcelona con Juan II. Gabriel Cerdà se embarcó hacia Catalunya desde el puerto de Sóller el 20 de marzo de $1472^{64}$, y no regresó a Mallorca hasta el 13 de septiembre del año siguiente ${ }^{65}$. El topónimo "Catalunya" utilizado por las fuentes es demasiado amplio, sin que se pueda determinar el lugar concreto donde recaló, en parte porque tampoco se conoce el

56. Muchos estudiantes mallorquines iniciaban sus estudios en Lleida y luego obtenían el grado en las universidades italianas. Otros conseguían el bachillerato en Lleida y se doctoraban en la península Itálica. Planas Rosselló 2000, pp. 470-471. Finalmente, otros muchos optaban por cursar sus estudios directamente en Italia, puesto que tenían que afrontar los riesgos marítimos en cualquier caso. Ramis Barceló 2014, p. 58.

57. Lladonosa i Pujol 1970, p. 99. Jaume Mas y su compañero, llamado Espill, habían sido detenidos por haber herido a otro estudiante dentro de una iglesia. El oficial del obispo exigió la entrega de los dos estudiantes, que eran clérigos simples tonsurados, pero los paers se negaron a ello, lo que desembocó en una lucha en la plaza de la Suda, a la cual también se sumaron varios estudiantes del Colegio de la Asunción. Lladonosa i Pujol 1988, pp. 57-59.

58. Lladonosa i Pujol 1976, pp. 37-38. Este colegio había sido fundado en Lleida por Domènec Pons para alojar doce clérigos pobres. Sin embargo, no todos sus miembros eran pobres en el sentido económico, sino que también se incluían los hijos de los ciudadanos que no habían heredado predios o bienes suficientes como para conservar su ciudadanía. Lladonosa i Pujol 1970, p. 68.

59. Lladonosa i Pujol 1970, pp. 18-19. Véase también Lladonosa i Pujol 1945.

60. ACM, Mensa Capitular, 2769, f. 46v.

61. ACM, Mensa Capitular, 2770, f. 34v

62. ACL, AC_0051 (Actas Capitulares, 1457-1467), f. 252r.

63. ACL, AC_0051 (Actas Capitulares, 1457-1467), f. 259v.

64. ACM, Mensa Capitular, 2776, f. 30v.

65. ACM, Mensa Capitular, 2777, f. 31r. 
objetivo del viaje. Sin embargo, sí que se puede saber que entre el 29 de marzo ${ }^{66}$ y el 27 de agosto de $1473^{67}$ estaba en Lleida. Quizá solucionó primero algunos asuntos en Barcelona y luego pasó cinco meses en la sede de su canonicato, un hecho que, como se ha ido viendo, era bastante habitual. En cualquier caso, no fue este el último viaje de Cerdà a la ciudad del Segre, pues la continuó visitando hasta el mes de junio de $1480^{68}$.

La ciudad de Roma, centro neurálgico de la Iglesia católica ${ }^{69}$, tenía que ser necesariamente uno de los principales destinos de los viajes realizados por los canónigos de Mallorca, sobre todo antes de la dependencia mallorquina del arzobispado de Valencia impuesta por Alejandro VI en 1492. Además, la Curia Pontificia por sí misma ya constituía un polo de atracción, sin importar el lugar donde residiera en cada momento ${ }^{70}$. Son muchas las referencias a periplos desde Mallorca hacia la ciudad del Tíber, aunque los barcos debían atracar en los puertos de Ostia o de Civitavecchia, situado este a pocos quilómetros al noroeste de Roma. También Gabriel Cerdà se embarcó hacia la península Itálica el 31 de julio de $1458^{71}$, esto es exactamente una semana antes de la muerte de Calixto III, acaecida el 6 de agosto de aquel mismo año. El canónigo no regresó a Mallorca hasta finales de mayo de 1461, y lo hizo en una nave procedente de Barcelona ${ }^{72}$. La cronología de este viaje coincide también con la muerte de su tío, el cardenal Antoni Cerdà, que sucedió en Roma el 12 de septiembre de $1459^{73}$. En cualquier caso, parece ser que este hecho habría sido más bien casual y que el motivo del viaje de Gabriel Cerdà no tendría nada que ver con la salud del purpurado; de hecho, entre el 15 de noviembre de $1458^{74}$ y el 27 de junio de $1459^{75}$ Gabriel Cerdà estuvo en Lleida. Por tanto, tal como haría en su primer viaje a Nápoles, Cerdà querría aprovechar la influencia de su tío cardenal, o quizá simplemente se dirigió a Roma para solucionar algunos asuntos de carácter eclesiástico. Sea como sea, antes de regresar a Mallorca, Gabriel Cerdà estuvo un mes en Lleida -entre el 1 de abril y el 2 de mayo de $1461^{76}$, para pasar luego a Barcelona, donde embarcó hacia la isla.

Los viajes a la península Itálica, y muy especialmente los que condujeron los canónigos a la corte napolitana de Alfonso el Magnánimo, contribuyeron a la recepción de las corrientes humanistas en Mallorca ya durante el siglo XV ${ }^{77}$.

66. ACL, AC_0052 (Actas Capitulares, 1468-1475), f. 162r.

67. ACL, AC_0052 (Actas Capitulares, 1468-1475), f. 162r.

68. La última presencia de Gabriel Cerdà documentada hasta ahora en Lleida es del 27 de junio de 1480. ACL, AC_0053 (Actas Capitulares, 1475-1481), f. 164r.

69. Piñol Alābart 2013, p. 252.

70. Esta idea puede resumirse en el axioma de Sinibaldo Fieschi (futuro Inocencio IV) "Ubi papa, ibi Roma”. Rapp 1973, pp. 201-202.

71. ACM, Mensa Capitular, 2764, f. 39v.

72. ACM, Mensa Capitular, 2766, f. 39v.

73. Eubel 1894, p. 62.

74. ACL, AC 0051 (Actas Capitulares, 1457-1467), f. 79r.

75. ACL, AC_0051 (Actas Capitulares, 1457-1467), f. 97v.

76. ACL, AC_0051 (Actas Capitulares, 1457-1467), ff. 166r y 168v-169r.

77. Fueron muchos los mallorquines que visitaron la corte de Alfonso el Magnánimo. Estos mallorquines introducirían nuevas ideas en la isla. Barceló Crespí, Ensenyat Pujol 2000, p. 55. 
De hecho, los prebendados de la catedral mallorquina acabaron por conformar un círculo humanista ${ }^{78}$ que perduró entre la segunda mitad del siglo XV y los primeros años del $\mathrm{XVI}^{79}$. Los canónigos que formaban parte de este compartían una serie de elementos comunes, básicamente una sólida formación intelectual, en ocasiones adquirida en universidades italianas, de modo que poseían las capacidades y los recursos necesarios para conocer los movimientos culturales que se desarrollaban fuera del Reino de Mallorca ${ }^{80}$. Entre los principales eclesiásticos que formaban parte de este círculo humanista de la catedral de Mallorca se hallan los canónigos Esperandéu Espanyol y Arnau de Santacília, además del propio Gabriel Cerdà. Sin embargo, hay que indicar que el círculo humanista ya contaba con algunos precedentes dentro del cabildo catedralicio mallorquín, como el canónigo Francesc Eiximenis ${ }^{81}$ o el propio obispo de Mallorca, Juan García ${ }^{82}$ que, como se ha indicado, residió en la corte napolitana hasta la muerte del Magnánimo ${ }^{83}$. En otras palabras, Gabriel Cerdà participaría de las ideas humanistas que llegaron a Mallorca, a pesar de las dificultades para hablar de un humanismo insular; más bien hay que ver una introducción de ideas modernas procedentes de Italia que convivieron con la tradición medieval existente en Mallorca ${ }^{84}$. Cerdà estaba interesado en la antigüedad clásica, pero, exceptuando algunos elementos, como la posesión de un tapiz con la historia de Troya ${ }^{85}$ o la presencia de determinados libros en su biblioteca, no se tiene constancia de una intensa actividad cultural en este sentido.

Por otro lado, la ausencia de los canónigos requería el nombramiento de un procurador en Mallorca que se encargara de velar por sus intereses y, sobre todo, de percibir las rentas canonicales correspondientes. Los procuradores tenían que ser personas de plena confianza, por lo que frecuentemente eran otros canónigos, compañeros del cabildo, o beneficiados en la catedral mallorquina; en alguna ocasión un mismo presbítero podía ser procurador de dos o más prebendados. También son numerosos los casos de canónigos ausentes que establecieron como procuradores a miembros de su propia familia, generalmente padres o hermanos. Si no era posible un pariente tan cercano, se podía optar por otro familiar un poco más lejano, como un sobrino, pero con quien se pudiera confiar. Gabriel Cerdà fue cambiando de procurador a lo largo del ejercicio de su canonicato mallorquín. Así pues, durante los primeros años -documentado para el período 1451-1455designó como su procurador al canónigo Francesc Sala, que también lo era de su

78. El concepto de "círculo humanista" ha sido acuñado por Maria Barceló y Gabriel Ensenyat en varias publicaciones. La aportación más reciente a la cuestión es Barceló Crespí, Ensenyat Pujol 2013.

79. Ensenyat Pujol 2012, pp. 111-113.

80. Ensenyat Pujol 2013, p. 19.

81. Para la actividad cultural de Francesc Eiximenis, véase Barceló Crespí, Ensenyat Pujol 2000, p. 96.

82. Barceló Crespí, Ensenyat Pujol 2000, pp. 55-56.

83. Ensenyat Pujol 2012, pp. 111-113.

84. Barceló Crespí, Ensenyat Pujol 2000, pp. 19-24.

85. Ensenyat Pujol 2012, p. 112. 
tío Antoni Cerdà ${ }^{86}$. En 1464, en cambio, la procuración de Gabriel Cerdà pasó a un pariente, su sobrino Mateu Alber, futuro canónigo de la catedral de Mallorca ${ }^{87}$. Por su lado, en 1472 el procurador del cabildo catedralicio, Pere Llitrà, anotó en el libro correspondiente que entregó por orden de Gabriel Cerdà nueve libras a la honorable dona Begura, hermana del canónigo ${ }^{88}$. Pocos años más tarde, en 1477, el procurador de Gabriel Cerdà era el presbítero Joan Borràs, procurador que compartía con su sobrino Mateu Alber, ya canónigo ${ }^{89}$, del mismo modo que lo había hecho el propio Gabriel Cerdà al inicio de su canonjía. Por tanto, se observa como Cerdà buscó sus procuradores entre sus círculos más afines, gente de confianza que pudiera percibir los réditos que le correspondían por su dignidad eclesiástica.

\section{A MODO DE CONCLUSIÓN}

Este artículo ha querido presentar una aproximación a la movilidad de los canónigos mallorquines de la segunda mitad del siglo XV a través de la figura concreta de Gabriel Cerdà. Comparativamente, la movilidad de este canónigo no se diferenció demasiado de la de sus compañeros de cabildo catedralicio por lo que se refiere a los destinos, aunque el número de viajes fue bastante superior; de hecho, la mayor parte de los canónigos presentaba una movilidad escasa o nula, mientras Cerdà estuvo ausente durante muchos años. Así pues, para los treinta y siete libros de Mensa Capitular conservados que corresponden a la cronología en que ejerció su canonicato, Cerdà aparece en cuatro ocasiones como canónigo ausente, mientras su presencia durante toda la anualidad es documentada veinte veces. Durante los demás trece años, Cerdà estuvo algunos meses en Mallorca, mientras el resto del año lo pasaba fuera de la isla.

Este hecho se puede explicar por la circunstancia que Cerdà era canónigo en dos cabildos catedralicios diferentes, de modo que, a pesar de disfrutar del derecho de ausencia y del "privilegio de fructibus in absentia", optó por pasar temporadas en las dos sedes, aunque hubo una mayor presencia en Mallorca, probablemente por razones de carácter patrimonial ${ }^{90}$. En cualquier caso, la ausencia de los canónigos requería que hubiera un procurador que se encargara de velar por sus intereses y que percibiera las rentas correspondientes. Gabriel Cerdà nombró como procurador a su sobrino Mateu Alber, una práctica nada extraña entre los canónigos ausentes. En caso de no ser posible, un compañero de cabildo de confianza fue también una opción plausible.

86. Francesc Sala también recibía los frutos de la pavordia de Cerdà.

87. Mateu Alber solo percibió los frutos del canonicato; los de la pavordía los recibió el notario Joan Falcó. ACM, 2769, f. 47r.

88. ACM, Mensa Capitular, 2776, f. 30v.

89. ACM, Mensa Capitular, 2781, f. 30r.

90. Mas i Forners 2000. El de Gabriel Cerdà no fue un caso excepcional: los canónigos tendían a adquirir bienes rurales en las zonas de las cuales procedían en vistas a aumentar el patrimonio familiar. Suárez Beltrán 1986, p. 252. 
Uno de los fenómenos que se observa a nivel general es que los canónigos tendían a viajar más durante los primeros años de ejercicio del canonicato, esto es cuando eran más jóvenes y, por tanto, soportaban mejor las adversidades de un largo trayecto marítimo. A modo de ejemplo, Gabriel Cerdà accedió a la canonjía de Mallorca el 15 de agosto de $1450^{91}$. Durante los primeros diez años en esta prebenda no es hallado nunca un año entero en Mallorca, sino que se le documentan varios viajes, concretamente a Nápoles, Roma, Valencia y Lleida. Durante los siguientes veinte años aún realiza numerosos viajes, si bien a un ritmo más pausado y pasando algunas anualidades enteras en la catedral mallorquina, en la cual ejercía también la dignidad de sacrista. Finalmente, durante sus últimos doce años de vida se le encuentra en Mallorca ${ }^{92}$. Esta misma tendencia a viajar más durante sus primeros años de canonicato es habitual en otros canónigos mallorquines, aunque también se puede dar el caso de canónigos de Mallorca que optaron por marcharse de la isla los últimos años de su vida, una práctica, pero, que es más frecuente en el caso de los oriundos de otros territorios.

En definitiva, el estudio de los viajes de Gabriel Cerdà permite aproximarse al análisis de la movilidad de la gente de la Edad Media, en este caso de un colectivo específico, el de los canónigos de la catedral de Mallorca. Hay que tener en mente las dificultades para llevar a cabo los viajes durante el siglo XV, a pesar de las innovaciones que permitirían llegar hasta América el año siguiente a la muerte del canónigo Cerdà, y más en el caso de las islas, en que la necesidad de embarcarse y afrontar los peligros del mar $^{93}$ era una obligación. El hecho que los centros de poder político y religioso se hallasen fuera de Mallorca favorecía la elevada movilidad de los mallorquines, que, en ocasiones, necesitaban personarse en aquellos para solucionar ciertos asuntos. Por su parte, pero, los viajes también supusieron la introducción de nuevas ideas, en este caso procedentes de las corrientes humanistas de la península Itálica. El hecho que Gabriel Cerdà tuviera un tapiz que representaba un episodio de la Guerra de Troya indica el gusto del canónigo por el arte renacentista, que, muy probablemente, había conocido durante sus periplos.

\section{BIBLIOGRAFÍA}

Alvira Cabrer, Martín (1999), "Peregrinaciones medievales hacia Jerusalén, Roma y otras metas", XX Siglos, 10/41, pp. 83-100.

Andrade Cernadas, José Miguel (2014), “¿Viajeros o peregrinos? Algunas notas críticas sobre la peregrinación a Santiago en la Edad Media", Minius. Revista do Departamento de Historia, Arte e Xeografía, 22, pp. 11-31.

91. ACM, Mensa Capitular, 2758, f. 55v.

92. ACM, Mensa Capitular, 2789, sf.

93. No se olvide que uno de los argumentos presentados por los jurados de Mallorca para solicitar la fundación del Estudio General Luliano fue, precisamente, la voluntad de evitar los peligros del mar, infestado de corsarios. Lladó i Ferragut 1973, p. 39. 
Argilés, Caterina (2010), Una ciutat catalana en època de crisi: Lleida, 13581500. El Treball, els salaris, la producció agrícola i els preus a través dels llibres d'obra de la Seu Vella, Lleida.

Barceló Crespí, Maria; Ensenyat Pujol, Gabriel (2000), Els nous horitzons culturals a Mallorca al final de l'Edat Mitjana, Palma.

Barceló Crespí, Maria; Ensenyat Pujol, Gabriel (2013), Clergues il·lustrats. Un cercle humanista a l'entorn de la Seu de Mallorca (1450-1550), Palma.

Beltran Perelló, Maria Esperança (2008), "La Col·lecció Pascual i les relacions entre el Regne de Mallorca i el món musulmà mediterrani”, Barceló Crespí, Maria, El Regne de Mallorca: cruilla de gents $i$ de cultures (segles XIII-XV), Palma, pp. 179-188.

Bover de Rosselló, Joaquín María (1838), Memoria biográfica de los mallorquines que se han distinguido en la antigua y moderna literatura, Palma.

Cateura Bennàsser, Pau (2000), "Mundos mediterráneos: el reino de Mallorca y el sultanato mameluco (siglos XIII-XV)", Espacio, Tiempo y Forma. Serie III, Historia Medieval, 13, pp. 85-101.

Ensenyat Pujol, Gabriel (2012), "Humanisme i Renaixement a Mallorca: noves dades, nova periodització", eHumanista/IVITRA, 1, pp. 99-127.

Ensenyat Pujol, Gabriel (2013), "Otoño medieval y humanismo eclesiástico en Mallorca”, Escartí, Vicent Josep, Escribir y persistir. Estudios sobre la literatura en catalán de la Edad Media a la Renaixença, Los Ángeles-Buenos Aires, 1, pp. 16-39.

Eubel, Konrad (1894), Hierarchia catholica Medii Aevi, sive Summorum pontificum, S. R. E. cardinalium, ecclesiarum antistitum series ab anno 1431 usque ad annum 1503 perducta e documentis tabularii praesertim Vaticani collecta, digesta, edita per Conradum Eubel, Ratisbona.

Furió, Antoni (1852), Episcopologio de la Santa Iglesia de Mallorca, Palma.

Guiral-Hadziiossif, Jacqueline (1989), Valencia, puerto mediterráneo en el siglo $X V$ (1410-1525), Valencia.

Igual Luis, David (1997), "Comercio e industria en las ciudades mediterráneas", Revista d'història medieval, 8, pp. 373-394.

Igual Luis, David (2006-2008), “Operadores económicos y espacios de comercio en el Mediterráneo occidental (siglos XIII-XV)", Anales de la Universidad de Alicante. Historia medieval, 15, pp. 189-214.

Lladó i Ferragut, Jaume (1973), Historia del Estudio General Luliano y de la Real y Pontificia Universidad Literaria de Mallorca, Palma.

Lladonosa i Pujol, Josep (1945), El sitio de Lérida de 1464 en tiempos de Juan II de Aragón, Lleida.

Lladonosa i Pujol, Josep (1970), L’Estudi General de Lleida del 1430 al 1524, Barcelona.

Lladonosa i Pujol, Josep (1976), Relacions entre Mallorca i Lleida a l'època medieval, Barcelona.

Lladonosa i Pujol, Josep (1988), Anecdotari de l'Estudi General de Lleida (12971717), Lleida. 
López, María Dolores (2004), "Mallorca y el Magreb en la Baja Edad Media", Trillo, Carmen, Relaciones entre el Mediterráneo cristiano y el norte de África en época medieval y moderna, Granada, pp. 87-225.

Macaire, Pierre (1986), Majorque et le commerce international (1400-1450 environ.), Lille.

March Ques, Joan Francesc; Rosselló Vaquer, Ramon (1981), Història de Santa Margalida. Volum primer. De la Prehistòria al segle XVI, Santa Margalida.

Martí Sentañes, Esther (2008), "Incidència i relació dels síndics eclesiàstics de Lleida amb les corts medievals", Bertran i Roigé, Prim; Fité i Llevot, Francesc, Arrels cristianes. Presència i significació del cristianisme en la història i la societat de Lleida. Volum II. Temps de consolidació. La baixa Edat Mitjana (segles XIII-XV), Lleida, pp. 131-138.

Mas i Forners, Antoni (2000), “De pagesos a cavallers: l'extracció social i el patrimoni del cardenal Antoni Cerdà i del canonge Gabriel Cerdà (segles XVXVI)", Barceló Crespí, Maria, Al tombant de l'edat mitjana. Tradició medieval i cultura humanista, Palma, pp. 437-450.

Meloni, Giuseppe (1977), "Contributo allo studio delle rotte e dei commerci mediterranei nel Basso Medioevo", Medioevo. Saggi e Rassegne, 3, pp. 117-130.

Navarro Sorní, Miguel (2005), "Calixto III, papa valenciano", Callado Estela, Emilio, Valencianos en la historia de la Iglesia, Valencia, pp. 79-104.

Novoa, Feliciano (2003), De Finisterre a Jerusalén: Egeria y los primeros peregrinos cristianos, Santiago de Compostela.

Oldenbourg, Zoé (1965), Les Croisades, París.

Ortega Villoslada, Antonio (2008), El Reino de Mallorca y el mundo atlántico (1230-1349), A Coruña.

Ortega Villoslada, Antonio (2013), "Navegación mediterránea por el Atlántico. El caso de Mallorca (1230-1349)", Minius. Revista do Departamento de Historia, Arte e Xeografia, 21, pp. 45-60.

Piñol Alabart, Daniel (2013), "Notarios catalanes en Roma: los notarios matriculados en el Archivo de la Curia (1508-1671)", Historia, Instituciones, Documentos, 40, pp. 251-302.

Piqué Badia, Joan Ramon (2002-2003), "El naixement i la consolidació del Capítol catedralici lleidatà. La Seu Vella, 1168-1540", Seu Vella, 4, pp. 397-443.

Planas Rosselló, Antonio (2000), "Los estudiantes mallorquines en Italia y el humanismo jurídico”, Barceló Crespí, Maria, Al tombant de l'edat mitjana. Tradició medieval i cultura humanística, Palma, pp. 469-482.

Postan, Michael (1978), Medieval trade and finance, Cambridge.

Ramis Barceló, Rafael (2014), "Peregrinatio academica: legistas y canonistas de la Corona de Aragón en las universidades italianas durante el Renacimiento", Miscellanea Historico-iuridica, 13/1, pp. 35-66.

Rapp, Francis (1973), La Iglesia y la vida religiosa en Occidente a fines de la Edad Media, Barcelona. 
Ruzafa García, Manuel (1994), "Comercio internacional, regiones, desarrollo y subdesarrollo en Europa: un debate sobre el modelo de historia económica bajomedieval", Revista d'història medieval, 5, pp. 173-180.

Salicrú i Lluch, Roser (2008), "Mallorquins a Granada, granadins a Mallorca. Granada i Mallorca en les rutes mediterrànies i atlàntiques de la baixa edat mitjana”, Barceló Crespí, Maria, El Regne de Mallorca: cruïlla de gents i de cultures (segles XIII-XV), Palma, pp. 115-134.

Sevillano Colom, Francisco (1968-1972), “De Venecia a Flandes (vía Mallorca y Portugal)", Bolletí de la Societat Arqueològica Lul-liana, 33, pp. 1-33.

Simbula, Pinuccia Franca (1993), Corsari e pirati nei Mari di Sardegna, Cagliari.

Suárez Beltrán, Soledad (1986), El Cabildo de la catedral de Oviedo en la Edad Media, Oviedo.

Tangheroni, Marco (1996), Commercio e navigazione nel Mediterraneo, RomaBari.

Tate, Robert Brian (1955), Joan Margarit i Pau, cardinal-bishop of Gerona. A biographical study, Manchester.

Tognetti, Sergio (2005), "Il ruolo della Sardegna nel comercio mediterraneo del Quattrocento. Alcune considerazioni sulla base di fonti toscane", Archivio Storico Italiano, 603, pp. 87-131.

Urgell Hernández, Ricard (2000), Mallorca en el segle XV, Palma.

Vaquer Bennàssar, Onofre (1991), "El comerç marítim de Mallorca a la segona meitat del segle XV", Randa, 29, pp. 105-119.

Vaquer Bennàssar, Onofre (1995), "Immigrants a Mallorca a la segona meitat del segle XV”, Bolletí de la Societat Arqueològica Lul-liana, 51, pp. 125-140.

Vaquer Bennàssar, Onofre (1997), "El comercio marítimo de Mallorca en el tránsito a la Modernidad”, Studia historica. Historia Moderna, 17, pp. 147-156.

Vaquer Bennàssar, Onofre (1999), "Immigrants a Mallorca a la segona meitat del segle XV”, Bolletí de la Societat Arqueològica Lul-liana, 55, pp. 353-362.

Vaquer Bennàssar, Onofre (2001), El comerç marítim de Mallorca. 1448-1531, Palma.

Villanueva Morte, Concepción (2008), "La presencia de valencianos y aragoneses en la documentación notarial cagliaritana del siglo XV", Anuario de Estudios Medievales, 38/1, pp. 27-63.

Fecha de recepción del artículo: enero de 2016

Fecha de aceptación y versión final: marzo de 2016 